\title{
The Present and Future of Observational Studies of Ocean Worlds
}

A white paper for the Planetary Science and Astrobiology Decadal Survey 2023-2032

Authors: Villanueva, Gerónimo L. (NASA-GSFC, geronimo.villanueva@nasa.gov, Phone: +1-301-286-1528, Conor Nixon (NASA-GSFC, conor.nixon@nasa.gov), Lucas Paganini (NASA-HQ, lucas.paganini@nasa.gov), Martin Cordiner (NASA-GSFC/CUA, martin.cordiner@nasa.gov), Stefanie Milam (NASA-GSFC, stefanie.milam@nasa.gov), Gordon Chin (NASA-GSFC, gordon.chin@nasa.gov)

Co-signatories: Gurwell (CFA), Crismani (CSUSB), Wright Knutsen (NASA -GSFC), Guzman Caloca (UMD), Allen (NASA-GSFC/USRA), Liuzzi (NASA-GSFC/AU), Kofman (NASA GSFC/AU), Renaud (NASA-GSFC/USRA), Floyd (NASA-GSFC), Walker (NASA-GSFC/UMD/WHOI), Neveu (NASA-GSFC/UMD), Hudson (NASA-GSFC), Bjoraker (NASA-GSFC), Shiblee Barua (USRA/NASA-GSFC), Imke de Pater (UC Berkeley), Sarah Horst (Johns Hopkins University)

Do the icy moons, Europa and Enceladus, host habitable conditions at submerged hydrothermal vents? Are there other ocean worlds with similar potential astrobiological significance? Thanks to the plentitude of recent discoveries of extremophile organisms, the limits for habitable conditions have greatly expanded, and the hypothesized subsurface oceans on these moons one of the most habitable niches in our Solar System.

Observational and remote-sensing studies can provide unique insights into the processes sustaining the activity on these bodies and the potential habitability of their sub-surface. Measuring the composition of the plumes will test for chemical diversity of internal "oceans", while by performing high-resolution mapping, we will be able to identify the regions of active release, and the processes responsible for the activity.

As we discuss in this paper, remote sensing with space and ground-based observatories, in coordination with in-situ remote sensing of these objects will reveal unique information regarding the processes acting on these astrobiologically relevant worlds, and the potential for habitability of their sub-surface oceans.

\section{Take away message}

- Remote sensing and spectroscopic measurements provide unique means for probing the composition and potential astrobiological significance of ocean worlds.

- Current high-resolution ground-based observations provide the most sensitive way to directly measure water (i.e., Keck and ALMA). Support for ground-based studies should be maintained and enhanced.

- Future space observatories will provide the needed sensitivity to further explore these worlds, and high-resolution spectroscopic instrumentation should be pursued.

- Future orbital in-situ remote sensing spectroscopic capabilities will permit to map and to globally quantify the chemical diversity of ocean worlds. Support for in-situ spectroscopic capabilities (high-resolution preferred) should be ensured. 


\section{An introduction to ocean worlds}

The satellites of Jupiter and Saturn that are now considered 'ocean worlds' were first observed not long after the invention of the telescope: the large moons of Jupiter by Galileo and Marius in 1610 and Titan by Huygens in 1655, while Triton was discovered soon after Neptune itself, in 1846 by Lassell. Little progress was possible on unraveling their actual nature until the space era, when finally the Voyager 1 \& 2 spacecraft (Stone 1977) made close flybys of all four giant planets in the decade from 1979 to 1989 , radically improving our understanding.

The first close-up imagery of the Jovian satellites (Smith et al. 1979a/b) revealed distinct differences between the four large moons. While lo was found to be hot and volcanic, Ganymede and Callisto exhibited a more typical cratered appearance seen on other similar-sized airless bodies of the inner solar system, such as the Moon and Mercury. Europa however was radically different, with a smooth appearance and very few features suggestive of impacts, indicating a much younger surface age. Similar surprises were found when the Voyagers reached the other outer planet systems. Titan was already known to have an atmosphere due to ground-based observations of Kuiper in 1944 that discovered methane (Kuiper et al. 1944), but close-up imaging revealed for the first time its exquisite photochemical haze layers (Smith et al. 1981).

Ground-based spectroscopy of Titan in the 1970s that had showed the presence of several hydrocarbons, but now the infrared spectrometer on Voyager 1 made the first detailed assay of its atmospheric composition (Hanel et al. 1981), showing the presence of nitriles, including $\mathrm{HCN}$ and $\mathrm{HC}_{3} \mathrm{~N}$, as well as $\mathrm{CO}_{2}$. The last major discovery made by the Voyagers pertaining to ocean worlds was the finding that Triton had both an atmosphere, and internal activity evidenced by surface geysers (Smith et al. 1989).

The next significant advance at Europa came with the Galileo mission in the 1990s, becoming the first orbiter of Jupiter. Close-up reconnaissance imaging of Europa's surface showed vast fragmented ice rafts, apparently pieces of a giant puzzle board that had been broken up, turned around and frozen back together in a jumbled confusion: these became known as 'chaos terrain(s)' (Greeley et al. 1998) (Fig. 1(b). By integrating this morphological information with that obtained by radio-tracking from the Galileo spacecraft, a possible ocean (or a low-density convective ice layer) of 80-170 km thickness is inferred to be present below Europa's icy crust (e.g., Khurana et al. 1998).

A similar spotlight fell on Enceladus after the arrival of the Cassini mission in 2004. Although Enceladus was the clearest candidate for an internal ocean, gravity and other measurements by Cassini have also led to predictions of internal oceans on Titan, Dione and possibly Mimas (less et al. 2012, Beuthe et al. 2016, Tajeddine et al. 2014). However, unlike Europa, the presence of a global sub-surface ocean is not suggested for Enceladus.

Instead, in Enceladus smaller non-continguous reservoirs containing pressurized liquid water beneath its southern pole have been suggested while strong shell librations (Hemingway \& Mittal 2019) suggest a global ocean. Using gravitational studies, less et al. established the depth of this reservoir to be 30-40 kilometers, and to extend to south latitudes of $\sim 50$ degrees. First in-situ measurements of the plumes, 
revealed a chemical composition similar to comets (Waite et al. 2006). Later results further revealed a great diversity of large organic compounds (e.g., Postberg et al. 2018), salts (Postberg et al. 2009) and non-volatile species (e.g., nanometer silica particles, Hsu et al.).

(a)

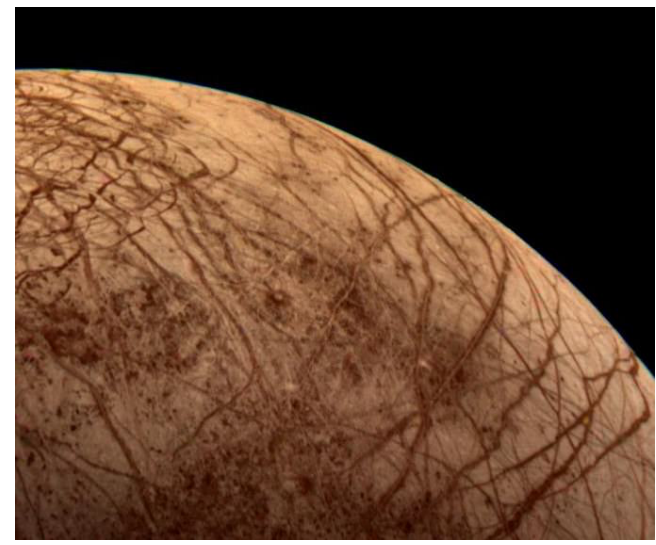

(c)

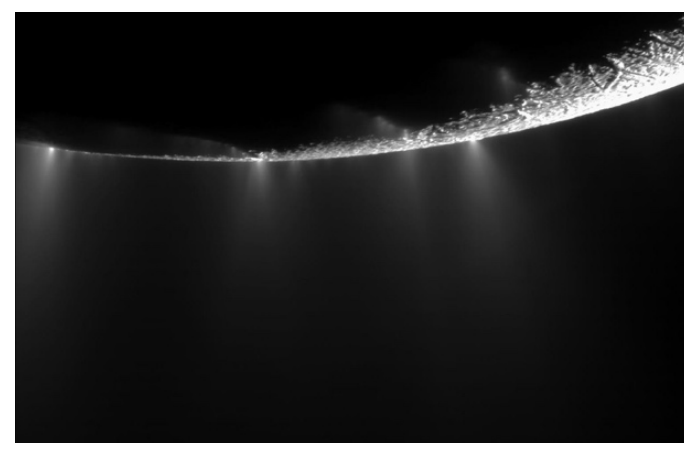

(b)

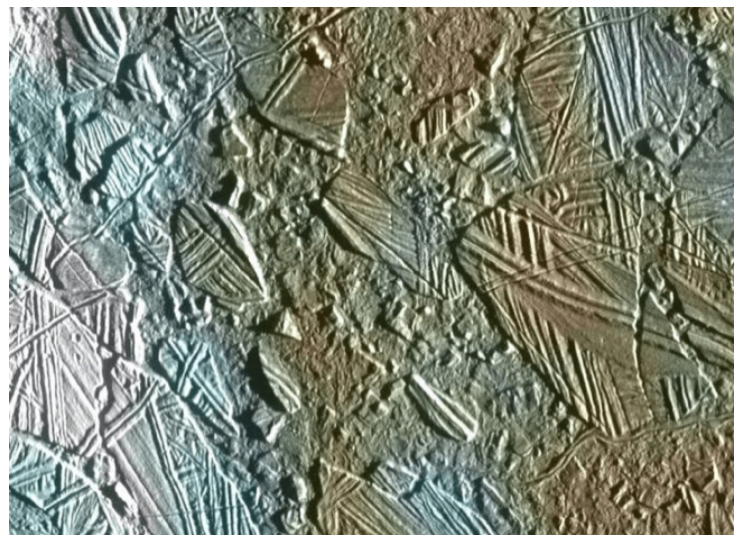

(d)

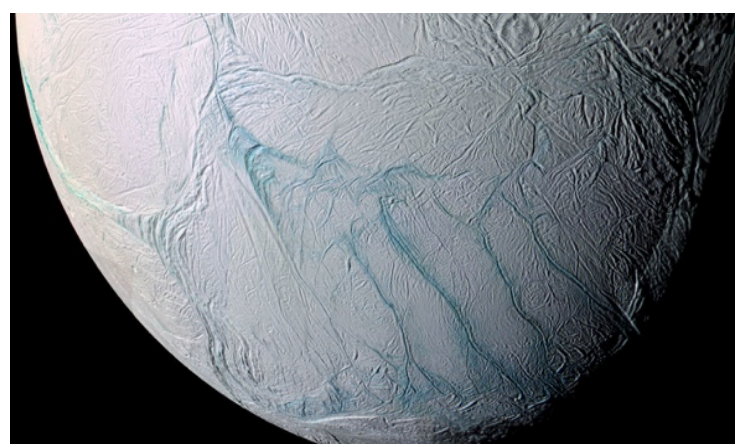

Figure 1. (a) Europa from Voyager 2, showing cycloid fractures (NASA/JPL) (b) Connemara chaos on Europa seen by Galileo (NASA/JPL/University of Arizona) (c) Enceladus jets seen by Cassini imaging system, 2010 (NASA/JPL/SSI) (d) Enceladus south pole, showing prominent fractures (blue) that are source of jets (NASA/JPL/SSI).

\section{Ocean worlds with ground-based IR/optical observatories}

Cassini discovered water plumes in Saturn's moon Enceladus in 2006 (e.g., Porco et al. 2006), and estimates have indicated that water release feeds the torus (e.g., Cassidy and Johnson 2010). Furthermore, in December 2012, UV observations with HST reported water vapor above Europa's south pole, drawing renewed attention to the satellite (Roth et al. 2014). Indeed, other Jovian moons like Ganymede and Callisto seem to have asymmetric water vapor atmospheres according to Herschel observations (Hartogh et al., in preparation). Ground-based observations are essential to aid these space-borne results. The high spectral and spatial resolution capabilities of these facilities present an alternative means to detect water directly. Most of these observations use heritage techniques that have resulted from decades-long observations of planetary atmospheres and comets (e.g., Mumma et al. 1996, Villanueva et al. 2015). 
Estimates of water vapor on Europa, either from exogenic or endogenic sources, have relied strongly on modeling efforts, yet confirmation from observational data has been somewhat incomplete. In 2012, ultraviolet observations with Hubble found simultaneous auroral emission features of hydrogen and oxygen in the southern hemisphere, at abundances $\left(>10^{29}\right.$ molecules $\left.\mathrm{s}^{-1}\right) 100-1000 x$ above the expected values resulting from exogenic effects. This was the first successful detection and characterization of plume content on Europa. These spectroscopic measurements of plume-like $\mathrm{H}$ and $\mathrm{O}$ emissions, however, have eluded confirmation despite numerous attempts, making detection of these features an elusive endeavor.

The key advantage of observations at infrared wavelengths is the ability to measure $\mathrm{H}_{2} \mathrm{O}$ (not $\mathrm{H}$ and $\mathrm{O}$ ), thereby providing a direct and independent assessment of water vapor on Europa's atmosphere. In the nearly collision-less environment, solar excitation of molecules (water and organics) results in emission of infrared photons through decay to the ground vibrational state, either directly (resonant fluorescence) or through branching into intermediate vibrational levels (non-resonant fluorescence), allowing high resolution spectroscopy to detect these signatures. Since 2016, groundbased observations of Europa targeted radiative excitation of water molecules via solar excitation in the 2-5.5 m wavelength range (Paganini et al. 2020), along with organics such as ethane, methanol, methane, hydrogen cyanide, formaldehyde, and ammonia (depending on the instrument configuration). Of their 17 observations, their results on 16 dates indicated no detection of emission features within sensitivity limits, thus establishing a rather quiescent state. The sensitive upper limits for water outgassing indicated column densities as low as $1.3 \times 10^{19} \mathrm{H}_{2} \mathrm{O} \mathrm{m} \mathrm{m}^{-2}$ and $2.6 \times 10^{19} \mathrm{H}_{2} \mathrm{O} \mathrm{m} \mathrm{m}^{-2}$ (3) in 2016 and 2017, respectively. One measurement, however, yielded an indication of spectral emission suggesting the presence of water vapor on 26 April 2016. The signal-to-noise ratio of the retrieved $\mathrm{H}_{2} \mathrm{O}$ spectrum (3.1) prevented establishing a definitive detection, yet co-addition of several spectral water lines and cross-correlation analysis of the observed spectra provided further statistical significance, corroborating the presumed water activity.

All individual detection strategies and wavelength regimes used to date have provided independent assessments that together convey the idea that observed features on Europa are transient and likely a result of plume activity (as suggested by the large number of non-detections in our survey and recent follow-up auroral measurements with HST). However, these conclusions are strongly driven -and hampered- by the sensitivity limits of current ground and space-based facilities, making detection of lower activity levels and proper characterization of Europa's atmosphere a complex endeavor with current technology. In the mid-2020s, the 30m-class telescopes (e.g., the Thirty Meter Telescope and Extremely Large Telescope) will provide the sensitivity and angular resolution needed to better characterize Europa, overcoming current limitations of $\sim 10 \mathrm{~m}$ class ground-based facilities in the infrared.

In 2030, two major missions, Europa Clipper and the Jupiter Icy Moons Explorer (JUICE) mission, will provide giant leaps in our understanding of Europa and other Jovian moons once they become online. Featuring unparalleled sensitivity and spatial resolution, these in situ remote-sensing observations are critical to obtain a closer assessment of active processes on Europa, including the complex interplay of release and sink mechanisms that shape its atmosphere as well as insights to the chemical fingerprints of fresh material 
released from the subsurface ocean through potentially active vents. In the meantime, observational data from ground-based observatories have served as unique means to constrain modeling efforts, improve observing strategies, and inform upcoming missions that promise to further unlock the secrets of Europa and other ocean worlds.

\section{Oceans worlds revealed with $\mathrm{mm} / \mathrm{sub}-\mathrm{mm} / \mathrm{far}-\mathrm{IR}$ observatories}

The radio, sub-millimeter and far infrared band (at wavelengths $>30$ micron) provides sensitive access to pure rotational transitions of dipolar, gas-phase molecules in planetary atmospheres and exospheres. The present generation of cutting-edge ground-based facilities (namely, the Atacama Large Millimeter/submillimeter Array; ALMA) is expected to be capable of detecting plume emission from ocean worlds such as Europa and Enceladus. To do so pushes the limits of our current observing capabilities, in particular, in terms of the required sensitivity, spatial resolution, weather, and instrumental calibration accuracy.

ALMA has excelled at the atmospheric sounding of denser icy moon atmospheres such as those of Titan, Pluto and Triton, demonstrating the power of heterodyne sub-mm spectroscopy for detailed chemical and physical studies. Major results include the detection of new (astrobiologically relevant) molecules (e.g. Palmer et al. 2017), measurement of isotopic ratios to help constrain atmospheric origins (Cordiner et al. 2018) and the retrieval of atmospheric temperatures and wind fields (e.g., Lellouch et al. 2019). Fundamental advances in our understanding of more tenuous atmospheres/exospheres surrounding other icy bodies are expected from sub-mm observations with current and future facilities.

Sub-mm observations of $\mathrm{H}_{2} \mathrm{O}$ in planetary atmospheres are extremely difficult using ground-based telescopes due to the Earth's atmospheric opacity, which precludes detection of the strongest lines of this key molecule. However, the $\mathrm{H}_{2} \mathrm{O}$ line at $183 \mathrm{GHz}$ (with upper-state energy of $205 \mathrm{~K}$, can be excited at the temperatures found in small body atmospheres), is accessible in the Band 5 atmospheric window, and provides a possible probe of outgassing and plume activity from icy moon surfaces. The unique, highresolution interferometric imaging capabilities of ALMA (with a spatial resolution up to 10 mas) can therefore provide detailed maps of atmospheric water vapor.

A key requirement of future $\mathrm{mm} / \mathrm{sub}-\mathrm{mm}$ wave facilities, for effective studies of plume chemistry, is the ability to spatially resolve the plumes (with sizes $\sim$ a few hundred $\mathrm{km}$ ), in order to distinguish them from background/ambient atmospheric emission, requiring beam sizes < 0.1". Obtaining sufficient sensitivity at this resolution is a challenge, even for ALMA, so a larger, more sensitive $\mathrm{mm} / \mathrm{sub}-\mathrm{mm}$ interferometer is recommended for development in the next decade.

A single, large-aperture telescope can also be considered, as long as the beam size is sufficiently small, so as not to excessively dilute already-weak plume signals. For example, a 100-m single-dish mm-wave telescope (with 3" beam at $1 \mathrm{~mm}$ wavelength) would be sufficient to detect Europa's $\mathrm{H}_{2} \mathrm{O}$ plume (with $\mathrm{N}\left(\mathrm{H}_{2} \mathrm{O}\right) \sim 1.5 \times 10^{16} \mathrm{~cm}-2$, assuming an excitation temperature of $100 \mathrm{~K}$ and a plume size $\sim 0.1$ "; Roth et al. 2014). Microwave 
emission from chemical tracers such as $\mathrm{HCN}, \mathrm{H}_{2} \mathrm{CO}$ and $\mathrm{H}_{2} \mathrm{~S}$, and potentially, more complex organic molecules, would also be detectable at a mixing ratio of $\sim 1 \%$ relative to $\mathrm{H}_{2} \mathrm{O}$, in a similarly-sized ocean world plume.

\section{The Future of Ocean Worlds with Next-Generation Space Observatories}

Next-generation observatories are currently being built, designed, and/or conceived for unprecedented sensitivity at multiple wavelengths as well as capabilities that will allow for new discoveries of these bodies. The James Webb Space Telescope (JWST) is the next great observatory currently slated to launch in 2021 . This $6.5 \mathrm{~m}$ diameter, passively cooled telescope will be the largest infrared observatory ever operated in space, covering wavelengths from 0.6-28.5 microns with low to medium spectral resolution, offering sensitivity that exceeds other infrared observatories by orders of magnitude. The JWST Guaranteed Time Observation (GTO) program includes these challenging measurements for bright objects such as Ceres, but will also give the preliminary glimpse at Mars, Titan, Europa, Enceladus, and Pluto. JWST will have access to multiple species of interest for detailed characterization and compositional studies of ocean worlds including: $\mathrm{H}_{2} \mathrm{O}, \mathrm{CO}, \mathrm{CO}_{2}, \mathrm{CH}_{4}, \mathrm{CH}_{3} \mathrm{OH}, \mathrm{OCS}$, etc. The pixel resolution of JWST will be able to map across the disk of satellites to determine surface variation and search for activity.
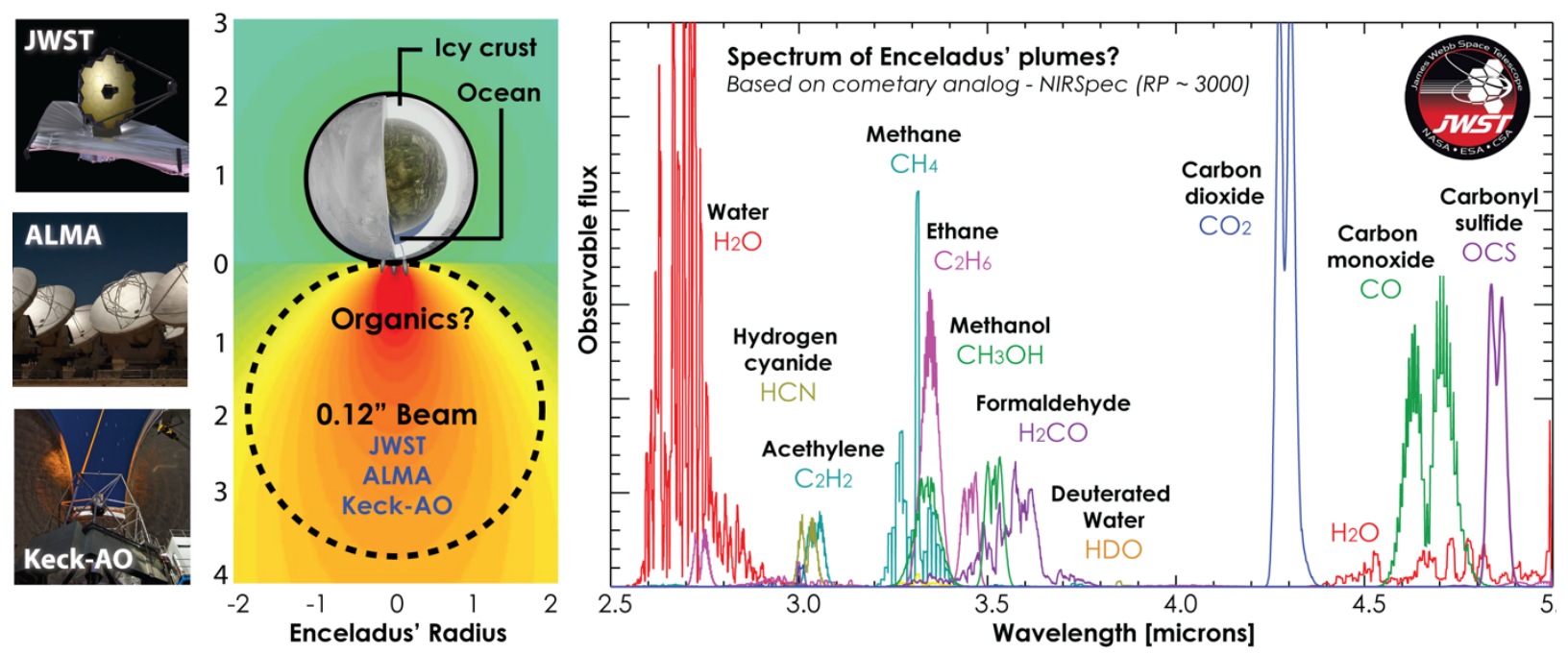

Figure 2: Ocean worlds can be sensitively probed by powerful space-based (e.g., JWST) and ground-based observatories (e.g., Keck-AO and ALMA). The plume structure is based on the model by Dong et al. 2011, while the model emission was synthesized considering typical cometary abundances, and employing the Planetary Spectrum Generator (Villanueva et al. 2018).

Studies for future space telescopes are currently being considered for the 2030's. These facilities will exceed the sensitivity and capability of all past/current/planned missions. Concepts that have been proposed include a large UV-Optical-NIR observatory (LUVOIR), the habitable exoplanet mission (Hab-Ex), the far infrared observatory (Origins 
Space Telescope), and an x-ray mission (Lynx). The first three will enable new science and realm of discovery for ocean worlds through significant enhancement not only in collection area, but also through new technology of the instrumentation. For example, LUVOIR will be able to image plumes across satellites as well as characterize them and determine abundances of water and other species with high precision. The Origins telescope will have access to water rotational and ro-vibrational lines to determine the abundance as well as isotopic ratios of volatiles. These new observatories will surpass the performance of previous missions such as Hubble, Spitzer, and Herschel space observatories. The access to the full sky, moving target tracking, wavelength coverage, and insurmountable sensitivity will reveal new secrets of ocean worlds that even orbiters may leave untold.

\section{Frontier instrumentation for ocean worlds}

The submillimeter is a prime spectral region for investigating these cold and tenous atmospheres/exospheres. A concept instrument being matured at GSFC, SELFI (Submillimeter Enceladus Life Fundamentals Instrument), aims to explore the water plumes ejected from Enceladus and 14 other species important in the context of habitability. SELFI is currently undergoing development as a MatISSE Project, while a related instrument, SSOLVE (Submillimeter Solar Observation of Lunar Volatiles Experiment,) is a DALI instrument aimed at highly sensitive lunar water detection using the Sun as a background source. In particular, submillimeter heterodyne receivers have proven to be powerful tools to explore different Solar System objects such as the Microwave Instrument for Rosetta Orbiter (MIRO) that has followed the evolving volatile expression of Comet 67/P from the outer solar system to its perihelion, and the planned Submillimeter Wave Instrument (SWI) for the Jupiter Icy Moon Explorer mission to explore emissions of Jupiter's moons.

Maturing and advancing these technologies has also impact for future instrumentation on space observatories. For instance, the Orbiting Astronomical Satellite for Investigating Stellar Systems (OASIS), a MIDEX concept, will be a 20-meter space observatory that will perform high spectral resolution $\left(R>10^{6}\right)$ observations at gigahertz to terahertz frequencies. OASIS will have $\sim 30 x$ the collecting area and $\sim 6 x$ the angular resolution of Herschel and complements the shorter wavelength capabilities of JWST. OASIS Science goals are highly complementary to the Origins Space Telescope and fills in a number of science gaps left by the descoped HERO instruments. With OASIS's large collecting area and suite of gigahertz to terahertz heterodyne receivers, it will have the sensitivity to explore the role water plays in these fascinating ocean worlds.

\section{References}

Beuthe et al., Geophysical Research Letters, vol. 43, no. 19, pp. 10088-10096 (2016); Cassidy et al., Icarus 201, 182-190 (2009); Cordiner et al., The Astrophysical Journal, bf 859, L15 (2018); Dong et al., Journal of Geophysical Research: Space Physics 116, 10204 (2011); Greeley et al., Icarus, vol. 135, no. 1, pp. 4-24, doi: 
10.1006/icar.1998.5969 (1998); Hanel et al., Science, vol. 212, no. 4491, pp. 192-200, doi: 10.1126/science.212.4491.192 (1981); Hsu et al. 2015, Nature 519, 207-210; less et al., Science, vol. 337, no. 6093, pp. 457-459, doi: 10.1126/science.1219631 (2012); Khurana et al. Nature 395, 777-780 (1998). https://doi.org/10.1038/27394; Kuiper, Astrophysical Journal, vol. 100, no. 3, pp. 378-383, doi: 10.1086/144679 (1944); Lellouch et al., Nature Astronomy, 3, 614 (2019); Mumma et al., Science 272, 1310 1314 (1996); Paganini et al., Nat Astron 4, 266-272 (2020); Palmer et al., Science Advances, Vol. 3, no. 7, e1700022, DOI: 10.1126/sciadv.1700022 (2017); Porco et al., Science. 311, 1393-1401 (2006); Postberg et al. 2009, Nature, Vol. 459, Issue 7250, pp 1098-1101; Postberg et al. 2018, Nature, Vol. 558, Issue 7711, p. 564-568; Roth et al, Science 343, 171-174 (2014); Smith et al., Science, vol. 206, no. 4421, pp. 927-950, doi: 10.1126/science.206.4421.927 (1979a); Smith et al., Science, vol. 204, no. 4396, pp. 951, doi: 10.1126/science.204.4396.951 (1979b); Smith et al., Science, vol. 212, no. 4491, pp. 163-191, doi: 10.1126/science.212.4491.163 (1981); Smith et al., Science, vol. 246, no. 4936, pp. 1422-1449 (1989); Stone, Space Science Reviews, vol. 21, no. 2, pp. 75-75 (1977); Tajeddine et al., Science, vol. 346, no. 6207, pp. 322-324 (2014); Villanueva et al., Science 348, 218-221 (2015); Villanueva et al, Journal of Quantitative Spectroscopy and Radiative Transfer, 217, 86-104 (2018); Waite et al., Science. 311, 1419-1422 (2006); Waite et al., 42nd LPSC. 42, 2818 (2011). 